20

\title{
Комплексное изучение особенностей поглощения сыворотки крови крыс с экспериментальным раком печени*
}

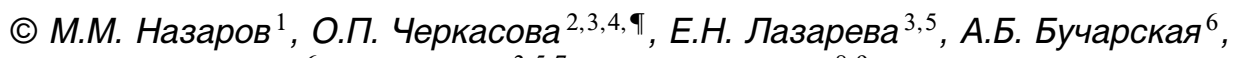 \\ Н.А. Наволокин ${ }^{6}$, В.В. Тучин ${ }^{3,5,7}$, А.П. Шкуринов ${ }^{8,9}$ \\ ${ }^{1}$ Национальный исследовательский центр „Курчатовский институт“, \\ 123182 Москва, Россия \\ ${ }^{2}$ Институт лазерной фризики Сибирского отделения РАН, \\ 630090 Новосибирск, Россия \\ ${ }^{3}$ Национальный исследовательский Томский государственный университет, \\ 634050 Томск, Россия \\ ${ }^{4}$ Новосибирский государственный технический университет, \\ 630073 Новосибирск, Россия \\ ${ }^{5}$ Саратовский национальный исследовательский государственный университет имени Н.Г. Чернышевского, \\ 410012 Саратов, Россия \\ ${ }^{6}$ Саратовский государственный медицинский университет им. В.И. Разумовского, \\ 410012 Саратов, Россия \\ ${ }^{7}$ Институт проблем точной механики и управления РАН, \\ 410028 Саратов, Россия \\ ${ }^{8}$ Московский государственный университет имени М.В. Ломоносова, \\ 119991 Москва, Россия \\ ${ }^{9}$ Институт проблем лазерных и инфрормационных технологий РАН — филиал ФНИЦ „Кристаллографрия и фротоника“, \\ 140700 Шатура, Россия \\ ؟e-mail: o.p.cherkasova@gmail.com
}

Поступила в редакцию 17.12.2018 г.

В окончательной редакции 13.02.2019 г.

Принята к публикации 26.02.2019 г.

\begin{abstract}
Методом импульсной терагерцовой (THz) спектроскопии в диапазоне частот $0.05-1.0 \mathrm{THz}$ проведены исследования сыворотки крови крыс в динамике развития экспериментального рака печени. Показано, что $\mathrm{THz}$ спектры пропускания образцов от здоровых животных и через 14 и 28 дней после имплантации клеток опухоли имеют подобную форму, но отличаются по амплитуде. Наблюдается изменение биохимического состава крови, в том числе уменьшение содержания белка, к 28 суткам эксперимента. Наблюдаемые изменения $\mathrm{THz}$ отклика объясняются уменьшением количества молекул белка и связанной с ними воды и коррелируют с изменениями показателя преломления в видимом и ближнем ИК диапазоне от 480 до $1550 \mathrm{~nm}$, измеренного с помощью многоволнового рефрактометра.
\end{abstract}

DOI: $10.21883 /$ OS.2019.06.47775.53-19

\section{Введение}

Высокое содержание воды в биологических жидкостях и подавляющем большинстве биологических тканей определяет характер их диэлектрического отклика в терагерцовом $(\mathrm{THz})$ диапазоне частот электромагнитного спектра [1]. Измеряемая характеристика в $\mathrm{THz}$ спектроскопии - это состояние самой воды, преобладающей в биологических образцах, которая, как известно, может находиться в свободном и связанном состояниях [2]. Изменения пропорций свободной и связанной воды, отличия во временах релаксации для каждого из этих состояний - именно это проявляется в $\mathrm{THz}$ диапазоне

\footnotetext{
* The 22nd Annual Conference Saratov Fall Meeting 2018 (SFM'18): VI International Symposium „Optics and Biophotonics“ and XXII International School for Junior Scientists and Students on Optics, Laser Physics \& Biophotonics, September 24-29, 2018, Saratov, Russia. https://www.sgu.ru/structure/fiz/saratov-fall-meeting/previousconferences/saratov-fall-meeting-2018
}

частот. Чувствительность к наличию свободной воды находится в диапазоне от 0.01 до $1 \mathrm{THz}$, поскольку характерная частота пика поглощения свободной воды соответствует $20 \mathrm{GHz}$, а его спектральная ширина простирается на 2-3 октавы в обе стороны [3]. В связи с этим для повышения чувствительности метода $\mathrm{THz}-$ спектроскопии необходимо расширить динамический диапазон THz-спектрометра в сторону низких частот.

Частотная зависимость комплексной диэлектрической THz-проницаемости воды и биологических тканей описывается релаксационными моделями, например двойной моделью Дебая с весьма близкими параметрами $[1,4-6]$. Релаксационный диэлектрический отклик тканей и растворов характеризуется отсутствием четко выраженных спектральных особенностей в THz-диапазоне частот $0.1-3 \mathrm{THz}$ В то же время чувствительность излучения к содержанию воды делает $\mathrm{THz}-$ спектроскопию и визуализацию привлекательными инструментами меди- 
цинской диагностики, использующими содержание воды в тканях и ее состояние в качестве информативных признаков для дифференциации [7-9].

Кровь представляет собой сложную систему, состоящую из плазмы и суспензированных в ней форменных элементов. Плазма крови содержит 90\% воды, около 6.6-8.5\% белков и других органических и минеральных соединений, которые являются промежуточными или конечными продуктами обмена веществ, переносимых кровью [10]. Различные составные компоненты крови оказывают значительное влияние на ее оптические свойства, причем их вклад может изменяться при развитии патологических состояний. Ряд авторов считает, что содержание в крови триглицеридов [11] и креатинина [12] оказывает значительное влияние на коэффициент поглощения и показатель преломления в диапазоне 0.2-1.0 THz. Количество эритроцитов в крови имеет отрицательную корреляцию с коэффициентом поглощения в $\mathrm{THz}$ области спектра $[6,11,13]$. Образование тромба при коагуляции крови оказывает значительное влияние на коэффициент поглощения при $200-270 \mathrm{GHz}$ [14]. Наиболее достоверно изучено влияние концентрации глюкозы в крови на ее оптические свойства в $\mathrm{THz}$ диапазоне частот $[15,16]$. В частности, при анализе крови 70 пациентов было продемонстрировано, что коэффициенты поглощения в $\mathrm{THz}$ области спектра и уровни глюкозы в крови имеют линейную зависимость [16].

Нами ранее было показано, что коэффициент поглощения и показатель преломления плазмы крови крыс с экспериментальным диабетом, измеренные в диапазоне частот $0.2-2.0 \mathrm{THz}$, достоверно ниже, а фаза коэффициента отражения выше этих показателей у здоровых животных [17]. Крысы с диабетом имеют содержание глюкозы в крови в 3.7 раза выше, чем у здоровых крыс. В предположении, что наблюдаемые спектральные изменения обусловлены изменениями состояния воды в плазме крови, было показано, что происходит уменьшение отношения $\Delta \varepsilon_{1} / \tau_{1}$ в 1.2 раза в главном релаксационном слагаемом в модели Дебая (см. формулу (5) ниже) в плазме крови крыс при увеличении концентрации глюкозы до 24 мМ [17].

Также было определено, что показатель поглощения плазмы крови крыс с тяжелым течением экспериментального диабета более чем в два раза меньше, чем это значение для крыс с неосложненным диабетом [18]. Содержание глюкозы и белка в плазме крови крыс, т.е. веществ, которые могут оказывать значительное влияние на состояние воды в $\mathrm{THz}$ диапазоне [19-21], статистически значимо не отличалось. Это говорит о том, что вклад в суммарное поглощение могут давать некоторые компоненты, которые содержатся в плазме крови при осложнениях диабета. Одними из таких компонентов могут быть продукты гликирования. Было показано значительное увеличение флуоресценции плазмы крови крыс с диабетом при возбуждении на длине волны $320 \mathrm{~nm}$, что свидетельствовало об увеличении количества конечных продуктов гликирования [22].
Было продемонстрировано уменьшение коэффициента поглощения плазмы крови мышей с перевитой карциномой Эрлиха в THz области спектра [23]. Авторы сделали предположение, что это отличие может быть вызвано изменениями в составе плазмы крови при развитии карциномы.

Таким образом, анализ литературы показывает, что изменение состава крови при патологиях может оказать значительное влияние на изменение оптических свойств крови в $\mathrm{THz}$ диапазоне частот, и это может быть использовано для создания новых методов экспрессдиагностики различных заболеваний. Особенный интерес представляет выявление онкологической патологии на ранних этапах, поиск объективных критериев для прогноза динамики развития заболевания и лечения. Холангиокарцинома (альвеолярный рак печени) - это злокачественная опухоль, которая образуется из мутировавших эпителиальных клеток желчных протоков. Количество заболеваний этим видом рака значительно увеличилось в последние годы [24]. Он характеризуется долгим периодом бессимптомного течения и обнаруживается, как правило, на последних стадиях течения болезни, когда прогноз излечения неудовлетворительный.

Настоящая работа посвящена изучению оптических характеристик сыворотки крови крыс в $\mathrm{THz}$ диапазоне частот в динамике развития холангиокарциномы, в выявлении отличий между образцами от здоровых и больных животных, сопоставлении данных с биохимическим составом крови и результатами рефрактометрии в видимом/ИК диапазонах длин волн.

\section{Материалы и методы}

\section{Методы измерения и обработки спектральной информации}

В работе использовали импульсный $\mathrm{THz}$ спектрометр, подробное описание которого приведено ранее в работах $[17,19,21]$. Особенность данного лабораторного спектрометра состоит в относительно высокой средней мощности THz-излучения - $10 \mu \mathrm{W}$ (от фотопроводящей многодипольной антенны и $1 \mathrm{~W}$ лазерного фемтосекундного излучения) [25], что позволяет просветить водный образец толщиной $0.5 \mathrm{~mm}$, вплоть до частоты $1 \mathrm{THz}$ (рис. 1). Поскольку мы исследовали вещества в виде растворов в воде, которая имеет сильное поглощение и дисперсию из-за релаксационного процесса в районе $10-100 \mathrm{GHz}[1,3]$, то и динамический диапазон $\mathrm{THz}$ спектрометра оптимизировался в низких частотах - до $0.05 \mathrm{THz}$. На рис. 1 расширенный диапазон спектрометра выделен темно-серым цветом по сравнению с обычным для импульсных $\mathrm{THz}$ спектрометров (рис. 1). На рис. 1, $a$ показан спектр мнимой части диэлектрической проницаемости $\varepsilon$ для трех составляющих водного раствора (для связанной воды по данным диэлектрической спектроскопии [26]), а на рис. $1, b$ - динамический 

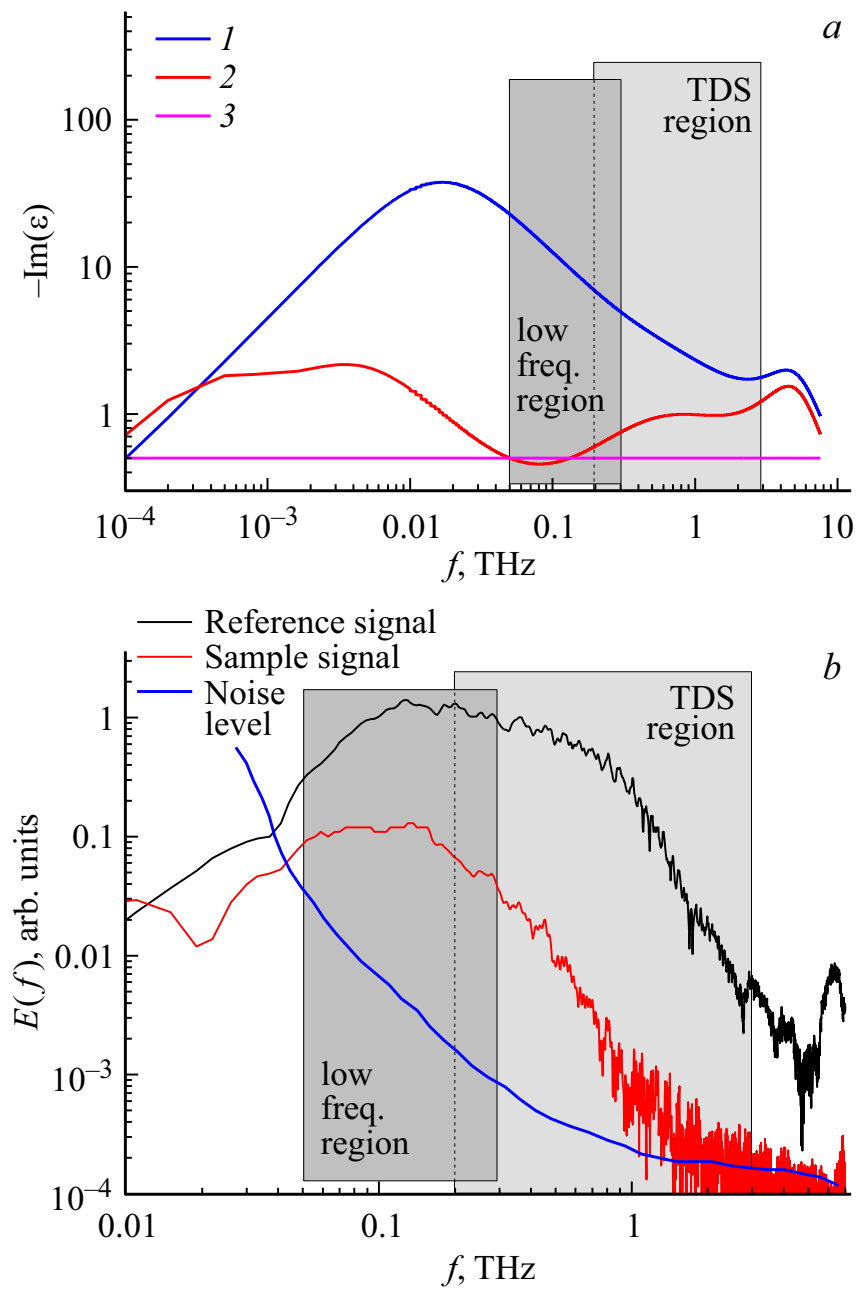

Pис. 1. (a) Спектр мнимой части диэлектрической проницаемости $\varepsilon$ для трех составляющих водного раствора: 1 спектр свободной воды (синяя линия); 2 - спектр связанной воды [26] (красная линия); 3 - спектр растворенного вещества (розовая линия); (b) динамический диапазон используемого $\mathrm{THz}$ спектрометра: спектр падающего излучения - черная линия; спектр прошедшего через $500 \mu \mathrm{m}$ воды сигнала $E(f)-$ красная линия; уровень шума - синяя линия. Низкочастотная часть $\mathrm{THz}$ спектра выделена темно-серым цветом, традиционный диапазон для импульсной спектроскопии - серым цветом.

диапазон используемой низкочастотной конфигурации спектрометра.

Для достаточного отношения сигнал/шум, при измерениях на крайне низких частотах (порядка $0.05 \mathrm{THz}$ ), использовалась долгая временная выборка, а именно от -20 до $40 \mathrm{ps}$ относительно центра $\mathrm{THz}$ импульса. Регистрация профилей сигнала $(A(t)$ на рис. 2) производилась с шагом по времени 0.05 ps. Для увеличения достоверности измерений производилось усреднение по 3 независимым измерениям для каждого образца сыворотки крови. Временная зависимость импульса электрического поля $\mathrm{THz}$ импульса $A(t)$ с помощью преобразования Фурье переводилась в его спектральное пред- ставление $E(f)$ (рис. $1, b)$, где $t$ - время, $f$ - частота. Экспериментальные образцы помещали в разборную кювету для жидкости (Bruker, США) с двумя одинаковыми окнами из полистирола. Показатель преломления окон постоянный в используемом диапазоне частот и равен $n_{c}=1.5$. Толщина слоя жидкости в нашем эксперименте составила $500 \pm 5 \mu \mathrm{m}$. Кювету устанавливали в коллимированный $\mathrm{THz}$ пучок. Отметим, что такая, относительно большая толщина слоя раствора необходима для обеспечения точности измерений в используемом частотном диапазоне. Толщина слоя задавалась штатным тефлоновым спейсером между окнами, одним и тем же для всех измерений. Все измерения проводили при комнатной температуре, $21 \pm 1^{\circ} \mathrm{C}$. Пропускание сыворотки крови $T_{w}(f)$ анализировали после нормировки на сигнал, прошедший через дистиллированную воду $E_{\text {water }}(f)$ в этой же кювете, измеряемый регулярно по очереди с сывороткой:

$$
T_{w}(f)=\frac{E_{\text {sample }}(f)}{E_{\text {water }}(f)} .
$$

Аналогичный подход к нормировке, позволяющий точнее измерять небольшие изменения в спектрах, был использован нами ранее при изучении плазмы крови крыс с диабетом $[17,18]$.

Для интегральной оценки отличия $\mathrm{THz}$ отклика сыворотки крови от отклика воды определялась полная амплитуда временного профиля $\mathrm{THz}$ импульса поля $A_{\text {sig }}$ (рис. 2). Эта величина также анализировалась после нормировки на опорный сигнал, прошедший через воду:

$$
T_{\mathrm{p}}=\frac{\left(A_{\max }^{\mathrm{sig}}-A_{\mathrm{min}}^{\mathrm{sig}}\right)}{\left(A_{\max }^{\mathrm{w}}-A_{\min }^{\mathrm{w}}\right)},
$$

где sig - образец, w - опорный сигнал от воды, $A_{\text {sig }}=A_{\max }^{\text {sig }}-A_{\min }^{\text {sig }}$ показан на рис. 2.

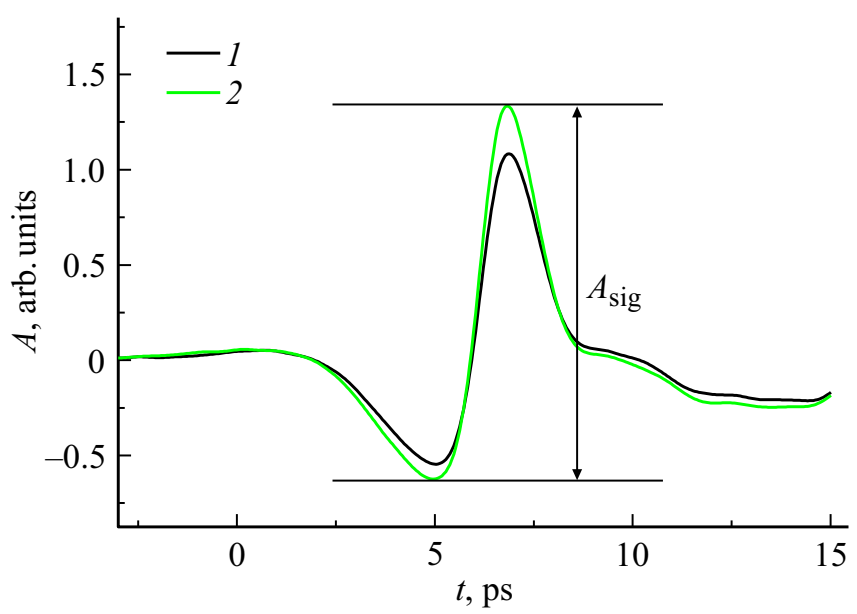

Рис. 2. Временной профиль $A(t)$ и пиковая амплитуда сигнала $A_{\text {sig: }} 1$ - прошедшего через $500 \mu \mathrm{m}$ слой воды (черная линия), 2 - прошедшего через $500 \mu \mathrm{m}$ слой образца сыворотки крови (зеленая линия). Нулевой момент времени соответствует максимуму импульса, прошедшего через слой воздуха. 
Учитывая, что спектр прошедшего сигнала расположен (по уровню 0.5 ) между 0.1 и $0.3 \mathrm{THz}$ (рис. $1, b$ ), величина $T_{\mathrm{p}}$ равна относительному коэффициенту пропускания $T_{\mathrm{w}}$, усредненному в этом диапазоне частот. Это примерно соответствует $T_{\mathrm{w}}(0.2 \mathrm{THz})$ и значениям $1.13-1.22$ для трех групп исследованных образцов (рис. 3 и 4 ).

Для пересчета спектра пропускания в спектры коэффициента поглощения и показателя преломления (при нормировке на падающий сигнал $E_{0}(f)$ ) использовали формулы Френеля и методы, описанные в работах $[17,19,21,27,28]$, а также формулы (8) и (9) (см. ниже) в случае нормировки на воду.

Существует однозначная связь измеряемой величины комплексного пропускания $T_{0}(f)=E(f) / E_{0}(f)$ с дисперсией диэлектрической проницаемости исследуемого вещества [19], а также соответственно с дисперсией его коэффициента поглощения и показателя преломления. В упрощенном виде частотные зависимости коэффициента поглощения $\alpha(f)$ и показателя преломления $n(f)$ могут быть представлены в следующем виде:

$$
\begin{aligned}
& \alpha(f)=-\frac{\ln \left(\left|T_{0}(f)\right| \frac{\left(n_{c}+n(f)\right)^{2}}{4 n_{c} n(f)}\right)}{d}, \\
& n(f)=-\arg \left[T_{0}(f)\right] \frac{c}{2 \pi f d}+1,
\end{aligned}
$$

где $d$ - толщина исследуемого образца, $c-$ скорость света. При этом $\varepsilon(f)=n^{*}(f)^{2}-$ связь комплексного показателя преломления образца с диэлектрической проницаемостью, где

$$
n^{*}(f)=n(f)+i \frac{\alpha(f) c}{2 \pi f} .
$$

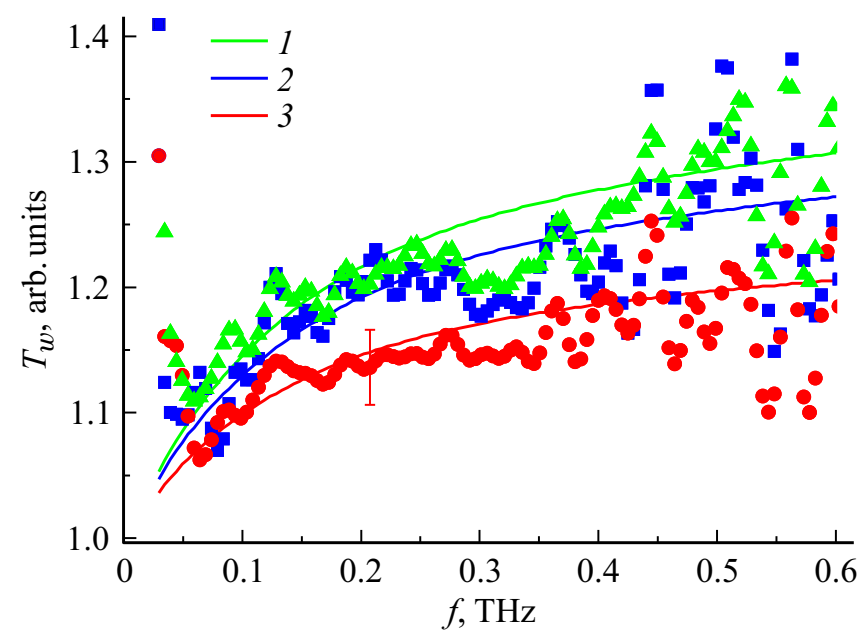

Pис. 3. Спектры пропускания сыворотки крови, нормированные на спектр пропускания кюветы с водой $-T_{\mathrm{w}}$. Точками показана средняя по каждой группе амплитуда пропускания: группа 1 - зеленые треугольники, группа 2 - синие квадраты, группа 3 - красные круги. Сплошные цветные линии модельные спектры с уменьшенной амплитудой $\Delta \varepsilon_{1}: 1-$ для группы 1,2 - для группы 2, 3 - для группы 3 .
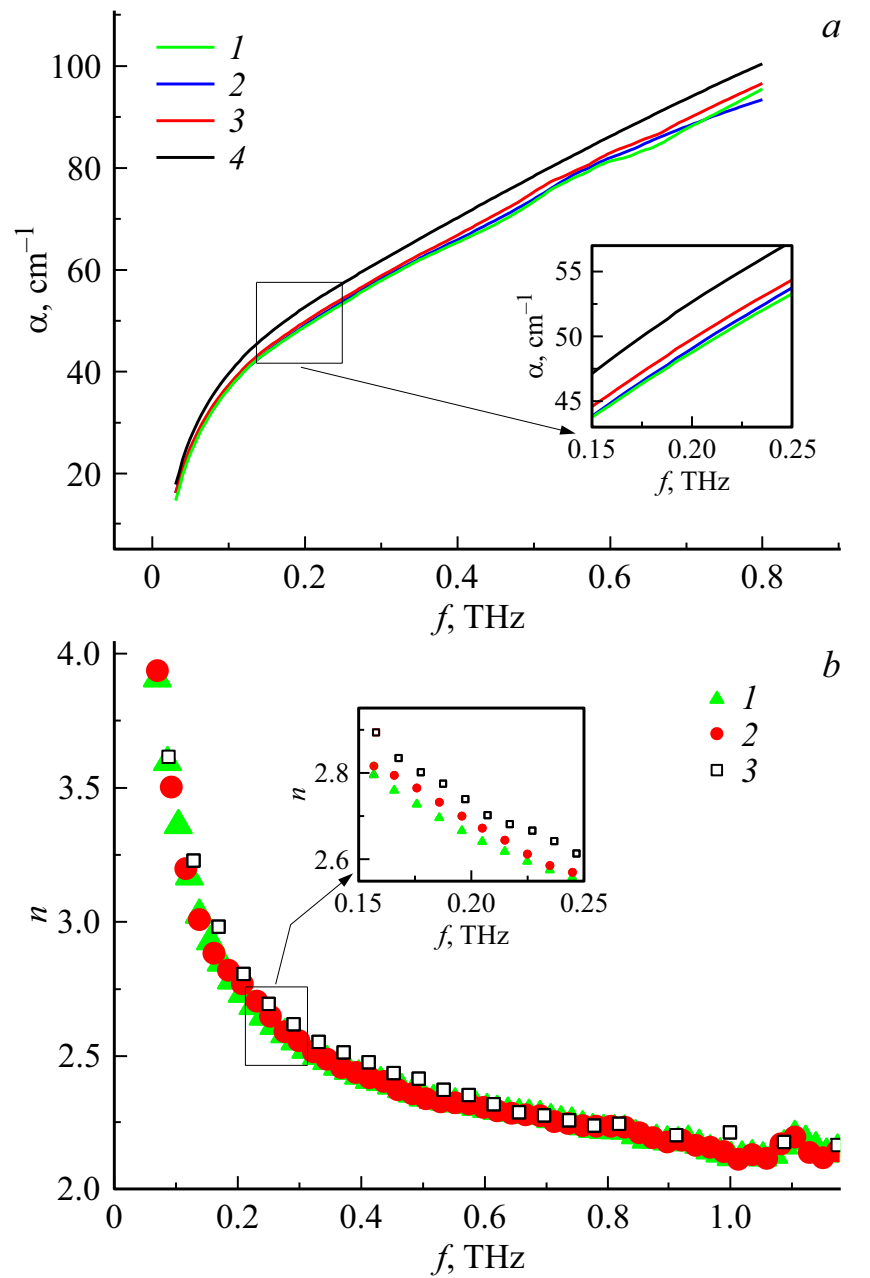

Рис. 4. (a) Спектры коэффициента поглощения для воды и образцов сыворотки крови: 1 - группа 1 , зеленая линия; 2 группа 2, синяя линия; 3 - группа 3 , красная линия; 4 вода, черная линия; $(b)$ спектры показателя преломления для образцов сыворотки крови: 1 - группа 1 (треугольники); 3 группа 3 (круги), 4 - вода (квадраты).

Множитель $\frac{\left(n_{c}+n(f)\right)^{2}}{4 n_{c} n(f)}$ в (3) нужен при низких частотах для учета потерь на отражение от границ воды. Отметим, что в данной работе мы используем коэффициент поглощения по амплитуде поля, а не по мощности, который в два раза больше и общепринят. Инструментальные ошибки, связанные с точностью определения толщины слоя раствора в кювете, дрейфом базовой линии, в итоге приводят к общей ошибке $5 \%$. Именно на такую величину расходятся результаты для $\varepsilon(f)$, пересчитанные из данных пропускания. На краях частотного спектра $(0.05-0.07$ и $0.8-1.0 \mathrm{THz})$ в силу малой величины сигнала ошибка/повторяемость ухудшается вплоть до 20\%. Приближенная формула (3) вносит ошибку менее $1 \%$ для вычисления величины поглощения для частот больше $0.05 \mathrm{THz}$ и слоя водного раствора не менее $0.5 \mathrm{~mm}$. 


\section{Характеристика животных}

Экспериментальное исследование было выполнено на белых беспородных половозрелых крысах-самцах по методике, описанной в работе [29]. Эксперименты на животных выполняли в соответствии с международными этическими нормами [30]. В работе моделировали развитие альвеолярного рака печени (холангиокарцинома, PC1), осуществляя введение $0.5 \mathrm{ml} 25 \%$ опухолевой взвеси в растворе Хэнкса подкожно в область лопатки. Животных выводили из эксперимента на 14 и 28 день после имплантации опухоли. Измеряли массу тела и размер опухоли. Истинная масса тела экспериментальных животных вычислялась при вычитании массы опухоли из общей массы тела. Забор крови проводился в специальные пробирки с коагулянтом. После $10 \mathrm{~min}$ центрифугирования отделяли сыворотку крови, которую использовали для проведения биохимического анализа и спектральных измерений.

Биохимические исследования проводились на полуавтоматическом анализаторе Clima MC-15 (RAL, Испания) с использованием наборов реагентов для определения общего белка, альбумина и глюкозы (Диакон-ДС, Россия).

Животные были разделены на 3 группы по 8 особей в каждой группе: группа 1 - контрольные здоровые животные; группа 2 - животные через 14 дней после привития опухоли; группа 3 - животные через 28 дней после привития опухоли. Основные характеристики животных приведены в таблице. Количество животных в каждой группе при проведении спектроскопических исследований составило: группа $1-8$, группа $2-4$, группа $3-6$ особей.

\section{Рефрактометрия}

Эти же образцы сыворотки крови были использованы для измерения показателя преломления в видимом и ближнем ИК диапазонах на многоволновом рефрактометре Аббе DR-M2/1550 (Atago, Япония) со стабилизацией температуры. Для селекции длины волны использовались узкополосные интерференционные фильтры 480 , $486,546,589,644,656,680,800,930,1100,1550 \mathrm{~nm}$. Погрешность измерений, вносимая прибором, составляла \pm 0.0002 .

\section{Статистический анализ данных}

Для обработки полученных в ходе исследований данных был использован пакет прикладных статистических программ SSPS-13.0. Проверку нормальности распределения значений в выборке проводили с помощью теста Колмогорова-Смирнова. Рассчитывали среднее арифметическое $(M)$, среднеквадратическое отклонения, в случае отличия распределения от нормального использовали медиану и квартили. Показатель достоверности различий $(P)$ определяли с использованием критериев
Стьюдента $(t)$ и Манна-Уитни в случае отличия распределения от нормального. Для формализации соответствия оптических параметров в видимой/ИК и $\mathrm{THz}$ областях спектра рассчитывали коэффициент корреляции Пирсона, который позволяет оценивать линейные отношения между двумя переменными.

\section{Результаты и обсуждение}

В динамике развития опухоли наблюдается статистически значимое уменьшение концентрации как общего белка, так и альбумина в сыворотке крови (таблица). Содержание альбумина в сыворотке крови крыс группы 3 (28 дней после имплантации опухоли) почти в два раза меньше, чем в крови здоровых крыс и крыс группы 2 (14 дней после имплантации опухоли). В $\mathrm{THz}$ спектрах пропускания были выявлены отличия между образцами для всех исследованных групп животных (рис. 3). Анализ наблюдаемых отличий проводили, сравнивая полученные экспериментальные спектры с пересчетом этих же зависимостей из модельной диэлектрической функции воды $\varepsilon(f)$ :

$$
\begin{aligned}
& \varepsilon(f, C)=\varepsilon_{\infty}+\frac{\Delta \varepsilon_{1}(C)}{1+i 2 \pi f \tau_{1}} \\
& +\frac{\Delta \varepsilon_{2}}{1+i 2 \pi f \tau_{2}}+\frac{A_{1}}{\omega_{01}^{2}-(2 \pi f)^{2}+i \gamma_{01} 2 \pi f}+\ldots
\end{aligned}
$$

Значения для параметров в (5) уточнялись для дистиллированной воды в соответствующем диапазоне частот для каждого из рассматриваемых процессов [19]. Так, для наиболее существенного процесса медленной дебаевской релаксации $\left(\Delta \varepsilon_{1}, \tau_{1}\right)$, который доминирует в диапазоне частот $1-100 \mathrm{GHz}$ (с центром при $20 \mathrm{GHz}$ ), значения выбираем из данных диэлектрической спектроскопии. Параметры быстрого дебаевского процесса $\left(\Delta \varepsilon_{2}, \tau_{2}\right)$ согласуем с данными импульсной $\mathrm{THz}$ спектроскопии. Для высокочастотного лоренцева слагаемого $\left(A_{1}, \omega_{01}, \gamma_{01}\right)$ данные выбираем из результатов ИК фурье-спектроскопии, так как колебательные процессы начинают проявляться от $4 \mathrm{THz}$ и выше. В итоге получаем следующий набор параметров: $\Delta \varepsilon_{1}=72 \pm 1, \tau_{1}=9.96 \pm 0.5 \mathrm{ps}, \Delta \varepsilon_{2}=$ $=1.66 \pm 0.03, \tau_{2}=0.24 \pm 0.01 \mathrm{ps}, A_{1}=31 \quad(\mathrm{THz} \cdot 2 \pi)^{2}$, $\omega_{01} / 2 \pi=5.3 \mathrm{THz}, \gamma_{01} / 2 \pi=7 \mathrm{THz}$; сумма амплитуд всех учтенных процессов определяет остаток от статической восприимчивости в виде $\varepsilon_{\infty}=2.6 \pm 0.2$. Приведенный набор значений оптимален для диапазона частот $0.05-2.5 \mathrm{THz}$ при температуре $21-24^{\circ} \mathrm{C}$. Рассчитанный с помощью данных значений из (5) спектр показан на рис. $1, a$ линией 1 .

Ранее нами была показана справедливость простой модели $\varepsilon(f)$ для эталонных растворов альбумина и глюкозы в воде [16,19-21]. Суть модели (5) состоит в том, что в $\mathrm{THz}$ диапазоне частот любой водный раствор биомолекул можно с достаточной точностью описать 
Содержание белка и глюкозы в плазме крови экспериментальных животных и масса опухоли

\begin{tabular}{c|c|c|c|c|c}
\hline Группа $(n=8)$ & Масса крысы, g & Масса опухоли, g & Общий белок, g/l & Альбумин, g/1 & Глюкоза, М/1 \\
\hline Группа 1 (здоровые животные) & $400-500$ & - & $83[67 ; 86.3]$ & $37[35.5 ; 39.3]$ & $5.2[4.9 ; 5.7]$ \\
\hline $\begin{array}{c}\text { Группа 2 } \\
\text { (через 14 дней после привития опухоли) }\end{array}$ & $150-200$ & 0.98 & $73[69.5 ; 77.3]$ & $39[36,5 ; 40,8]$ & $7.8^{*}[6.7 ; 8.9]$ \\
\hline $\begin{array}{c}\text { Группа 3 } \\
\text { (через 28 дней после привития опухоли) }\end{array}$ & $150-200$ & 26.27 & $57^{* *}[55 ; 64]$ & $21^{* *}[19 ; 23]$ & $5.4[5 ; 5.7]$
\end{tabular}

Примечание. Достоверность отличий: ${ }^{*} p \leq 0.05$ по сравнению с группой 1 ; ${ }^{* *} p \leq 0.01$ по сравнению с группой 1 .

диэлектрической проницаемостью от спектра воды с единственным изменением амплитуды первой, медленной дебаевской релаксации. Учет концентрации растворенного вещества состоит в том, что в $\mathrm{THz}$ диапазоне любой водный раствор биомолекул, что справедливо и для сыворотки крови, можно с достаточной точностью описать преимущественно уменьшением амплитуды „медленной“ компоненты дебаевской релаксации $\Delta \varepsilon_{1}$ при росте концентрации растворенного вещества $C$. При этом

$$
\Delta \varepsilon_{1}(C)=\Delta \varepsilon_{1}(1-C K) .
$$

Эмпирический коэффициент $K$ при $\Delta \varepsilon_{1}$ однозначно связан с концентрацией растворенного вещества $C$ и тем самым влияет на спектр диэлектрической функции итогового раствора. Нами ранее [19] было показано, для раствора альбумина в воде следует использовать $K=8 \cdot 10^{-4} \mathrm{ml} / \mathrm{mg}$. Для других компонент сыворотки величина $K$ имеет тот же порядок величины [1]. Упрощенные формулы (5) и (6) применимы в диапазоне частот $0.01-2.5 \mathrm{THz}$ в силу того, что образующаяся связанная вода имеет время релаксации $\tau_{1}$ вне указанного диапазона (рис. 1,a) и по сути выключает часть молекул воды из процесса медленной релаксации с доминирующим поглощением.

Для получения модельного спектра диэлектрической проницаемости сыворотки крови группы 1 (контрольные животные, содержание белка $83 \mathrm{~g} / 1)$ необходимо $\Delta \varepsilon_{1}$ умножить на 0.9; для группы 2 (содержание белка $73 \mathrm{~g} / 1)$ - на 0.91 и для группы 3, которая имеет низкий уровень белка $(57 \mathrm{~g} / 1)$, — на 0.93 (рис. 3). Физический смысл аппроксимируемого в модели параметра $(1-C K)=\Delta \varepsilon_{1, \text { water }} / \Delta \varepsilon_{1 \text {,solution }}$ - это объемная доля свободной воды в растворе. Оставшаяся часть объема занята связанной водой и молекулами растворенного вещества; обе эти компоненты имеют пренебрежимо малое поглощение по сравнению со свободной водой в исследуемом диапазоне $0.05-1.0 \mathrm{THz}$ (рис. 1,a) [1].

Для определения величины $(1-C K)$ измеренные спектры образцов сыворотки крови разных групп животных в виде $T_{\mathrm{w}}(1)$ сравнивали с модельным пропусканием, пересчитанным из диэлектрической проницаемости $\varepsilon(f)(5)$ и (6) как

$$
T_{\mathrm{w}}(f)=\exp \left\{-\frac{i\left[\varepsilon_{\mathrm{s}}(f)^{0.5}-\varepsilon_{\mathrm{w}}(f)^{0.5}\right] d 2 \pi f}{c}\right\} .
$$

Отметим, что приближенная формула (7) не учитывает отражений на границах водного раствора. Это занижает величину $T_{\mathrm{w}}$ по сравнению с (1) не более чем на 0.02 для частот ниже $0.1 \mathrm{THz}$ при используемых в работе параметрах. Наибольшие отличия в спектрах пропускания наблюдались для образцов группы 3 относительно группы 1: пропускание для образцов сыворотки крови животных группы 3, которые характеризуются статистически значимо меньшим содержанием белка в крови, было меньше, чем для образцов сыворотки крови здоровых крыс (рис. 3). Каждый образец независимо измерялся по три раза. Видно, что наиболее достоверный диапазон спектральных значений лежит в интервале от 0.07 до $0.5 \mathrm{THz}$.

Из спектров пропускания $T_{\mathrm{w}}(1)$, нормированных на воду, можно вычислить изменение коэффициентов поглощения и показателей преломления:

$$
\Delta \alpha=-\frac{\ln \left(\left|T_{\mathrm{w}}\right|\right)}{d}, \quad \Delta n=-\frac{\arg \left(T_{\mathrm{w}}\right) c}{2 \pi f d} .
$$

Тогда, хорошо зная частотную зависимость опорного спектра воды (5), можно без дополнительных упрощений, ошибок и шумов восстановить спектры поглощения и преломления исследованных растворов (рис. 4):

$$
\begin{gathered}
\alpha_{\text {solution }}(f)=\operatorname{Im}\left[\varepsilon(f)^{0.5}\right] \frac{2 \pi f}{c}+\Delta \alpha(f), \\
n_{\text {solution }}(f)=\operatorname{Re}\left[\varepsilon(f)^{0.5}\right]+\Delta n(f) .
\end{gathered}
$$

Из рис. 4 видно, что наибольшие отличия наблюдаются в спектре показателя поглощения по сравнению со спектром показателя преломления. Форма спектров воды и сыворотки крови совпадает, но наблюдается отличие в амплитуде. Именно для выявления слабых отличий между исследованными образцами мы использовали прием нормировки на сигнал, прошедший через воду, как описано выше. Отметим, что если не учитывать отражения (которые в первом приближении сокращаются) в формулах (1), (7)-(9), ошибка определения $\alpha$ и $n$ составляет менее $0.5 \mathrm{~cm}^{-1}$ и 0.02 соответственно, что пренебрежимо мало.

Зная диэлектрическую функцию исследованных образцов, можно рассчитать амплитуду коэффициента отражения [17] и в перспективе применять THz-спектроскопию для бесконтактной диагностики, как было опробовано 


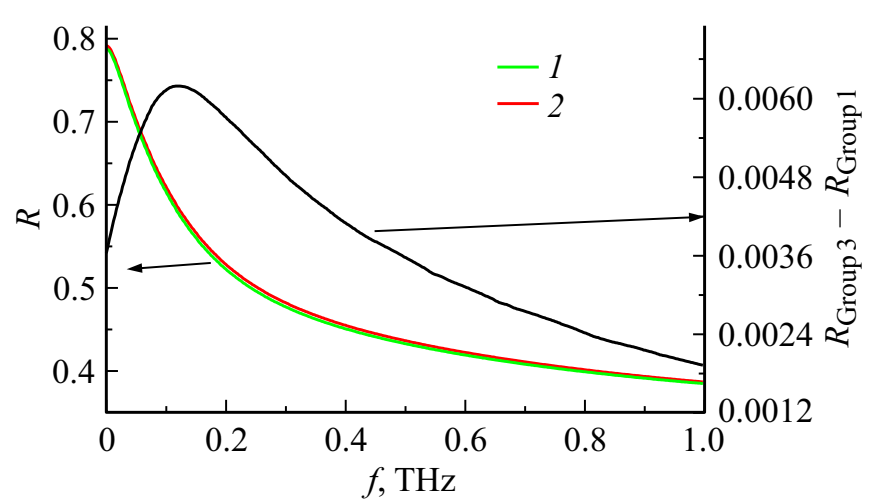

Рис. 5. Рассчитанные спектры отражения для образцов сыворотки крови группы 1 (зеленая линия) и группы 3 (красная линия). Черной линией показан разностный спектр отражения от образцов группы 1 и 3 (на графике 1 и 2 соответственно).

нами ранее для неинвазивного определения содержания глюкозы в крови [30]. Мы регистрировали изменение параметров сигнала отражения от кожи в зависимости от изменения содержания глюкозы в крови человека [31]. Для реалистичных концентраций компонент крови отличия в показателях преломления малы для нынешней точности эксперимента. Пересчитываем амплитуду отражения из модельной проницаемости как

$$
R=\left|\frac{\left(1-\varepsilon^{0.5}\right)}{\left(1+\varepsilon^{0.5}\right)}\right|
$$

Отличие в амплитуде отраженных сигналов для образцов из группы 1 и группы 3, пересчитанное как $R\left(\varepsilon_{\mathrm{Group} 3}\right)-R\left(\varepsilon_{\mathrm{Group} 1}\right)$, оценивается в $0.6 \%$ (рис. 5$)$. При этом максимальная чувствительность (в рамках модели (5) и (6)) ожидается в районе $0.1-0.3 \mathrm{THz}$. При повышении динамического диапазона $\mathrm{THz}$ устройств на 2 порядка можно будет регистрировать в будущем такое малое отличие в отражении и использовать данные результаты для неинвазивной визуализации в динамике развития патологии и контроля эффективности применяемых методов лечения.

Как видно из рис. 3-5, спектры широкополосные и бесструктурные. По ним видно отличие групп в среднем, но нельзя выделить оптимальную частоту для анализа отличий. Для интегральной оценки отличия $\mathrm{THz}$ отклика сыворотки крови от воды нами использовалась величина $T_{p}$, которая представляет собой пиковую амплитуду $\mathrm{THz}$ имульса (рис. 2), нормированную на пропускание воды. На рис. 6 показана величина $T_{\mathrm{p}}$ для каждого исследованного образца (рис. $6, a$ ) и приведена зависимость данной величины от содержания белка в образцах (рис. $6, b)$.

Как видно из рис. 6, наибольшие отличия в спектре пропускания наблюдаются между группой 1 (здоровых крыс) и крыс группы 3 (28 суток после имплантации опухоли), и эти отличия связаны с уменьшением содержания белка в сыворотке крови крыс в процессе развития опухоли.
В дополнение к результатам $\mathrm{THz}$ спектроскопии для тех же самых образцов был измерен показатель преломления в видимом и ближнем ИК диапазонах $(480-1550 \mathrm{~nm})$. На рис. 7 приведены значение показателя преломления на длине волны $589 \mathrm{~nm}$ по измеренным образцам и зависимость между показателем преломления на длине волны $589 \mathrm{~nm}$ и содержанием белка в сыворотке крови экспериментальных групп. Для других длин волн получена подобная зависимость.

На рис. 6 и 7 наблюдаются одинаковые тенденции в изменении оптических характеристик сыворотке крови крыс в динамике развития опухоли и их зависимость от концентрации наиболее изменяемого биохимического показателя - содержания белка в сыворотке крови.

Для количественной оценки связи оптических параметров в видимой/ИК и $\mathrm{THz}$ областях были рассчитаны коэффициенты корреляции Пирсона. Коэффициент корреляции между значениями параметра $T_{\mathrm{p}}$ и показателя преломления на длине волны $589 \mathrm{~nm}$ можно записать в

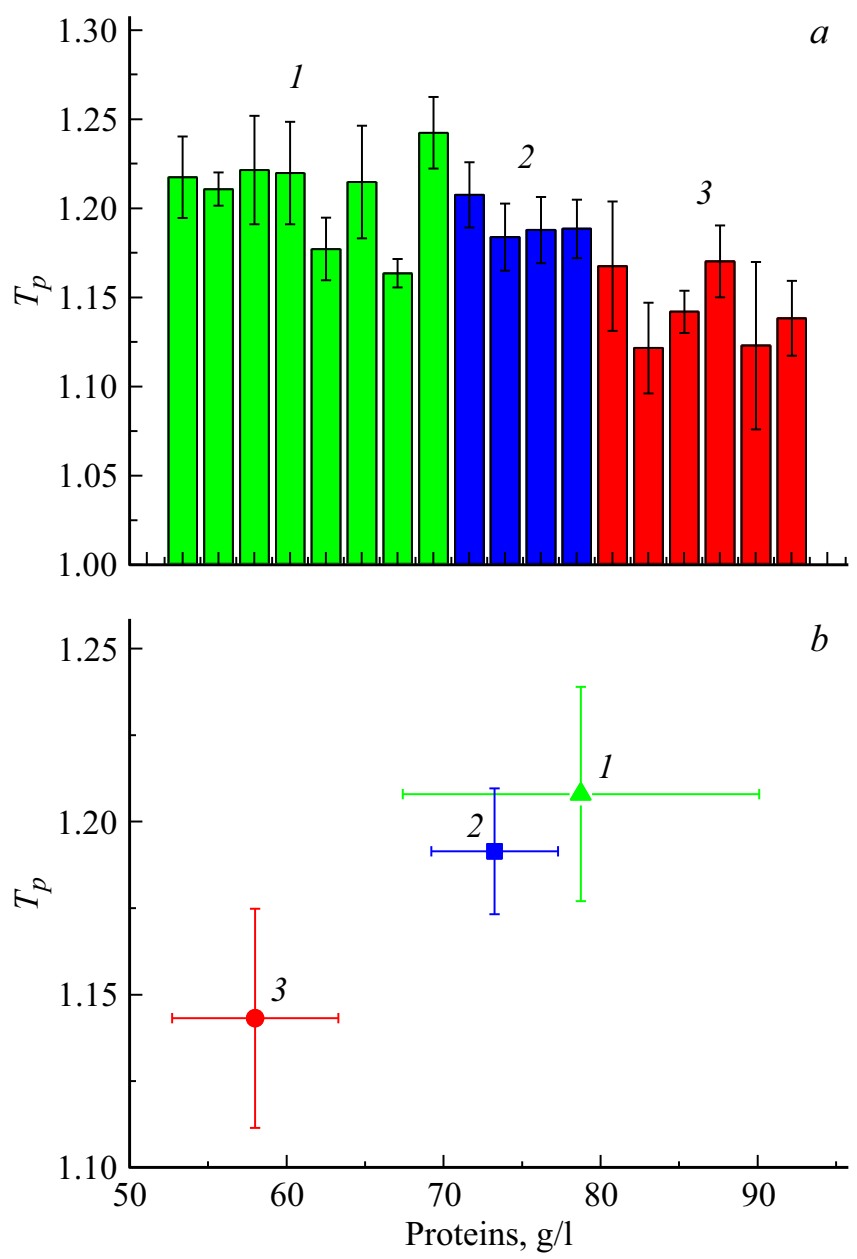

Рис. 6. (a) Значение $T_{\mathrm{p}}$ по образцам (усреднение по 3 пробам); (b) зависимость величины $T_{\mathrm{p}}$ от концентрации белка в образцах. Приведены средние данные по группам: 1 - группа 1 ; 2 - группа $2 ; 3$ - группа 3. 
виде

$$
\begin{aligned}
\rho_{n_{589}, T_{p}}= & \frac{\operatorname{cov}\left(n_{589}, T_{\mathrm{p}}\right)}{\sigma_{n_{589}}} \\
= & \frac{M\left\{\left[n_{589}-M\left(n_{589}\right)\left[T_{\mathrm{p}}-M\left(T_{\mathrm{p}}\right)\right]\right\}\right]}{\sigma_{n_{589}} \sigma_{T_{p}}},
\end{aligned}
$$

где $\sigma_{n_{589}}, \sigma_{T_{p}}-$ стандартные отклонения показателя преломления на длине волны $589 \mathrm{~nm}\left(n_{589}\right)$ и нормированной амплитуды пропускания в $\mathrm{THz}$ области $\left(T_{\mathrm{p}}\right)$ соответственно; $M\left(n_{589}\right)$ и $M\left(T_{\mathrm{p}}\right)$ - ожидаемое значение переменной; $\operatorname{cov}\left(n_{589}, T_{\mathrm{p}}\right)$ - ковариация.

Средний коэффициент корреляции, рассчитанный для каждой экспериментальной группы, составил $0.977 \pm 0.034$, что свидетельствует о высокой корреляции данных (рис. 8).

Уменьшение показателя преломления в видимой области при развитии онкологии у животных обусловлено уменьшением концентрации белка, так как концентрация
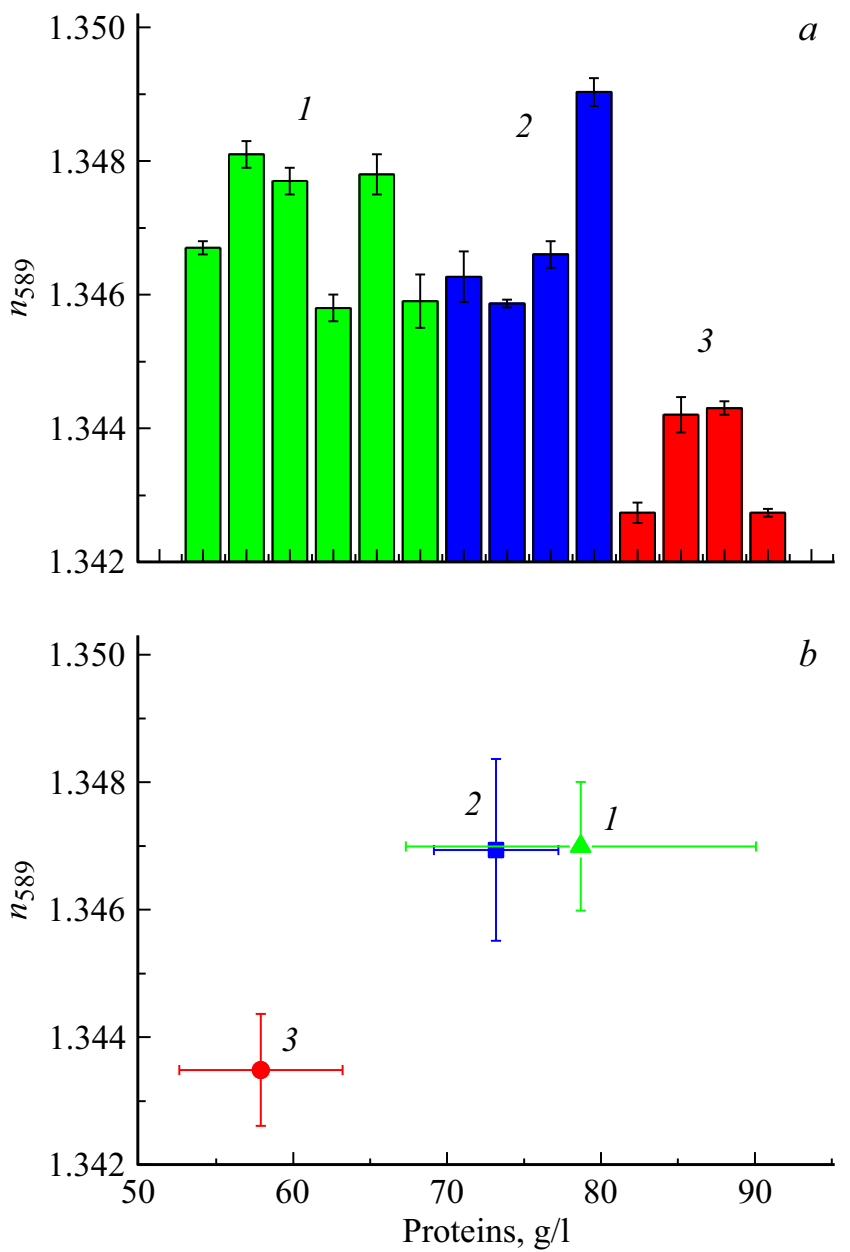

Pис. 7. (a) Значение показателя преломления на длине волны $589 \mathrm{~nm}$ по образцам (усреднение по 3 измерениям); (b) Зависимость между показателем преломления на длине волны $589 \mathrm{~nm}$ и содержанием белка в сыворотке крови экспериментальных групп. Приведены средние данные по группам: 1 - группа 1 ; 2 - группа $2 ; 3-$ группа 3.

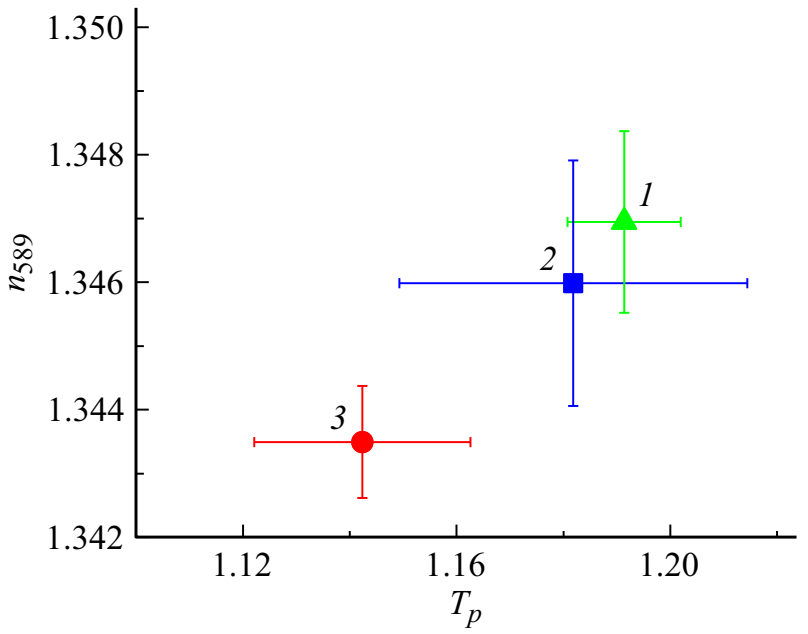

Рис. 8. Корреляция между показателем преломления на длине волны $589 \mathrm{~nm}$ и нормированной (на воду) амплитудой $\mathrm{THz}$ пропускания $T_{\mathrm{p}}$ сыворотки крови. Приведены средние данные по группам: 1 - группа 1 (треугольники), 2 - группа 2 (квадраты), 3 - группа 3 (круги).

белка в крови линейно связана с показателем преломления [32]. Высокая же корреляция между данными в $\mathrm{THz}-$ и видимой областях, например, позволяет восстановить дисперсионную зависимость в $\mathrm{THz}$ области по известным значениям показателя преломления в видимой области и по коэффициенту поглощения в $\mathrm{THz}$ области методом Крамерса-Кронига [33].

\section{Заключение}

В настоящей работе проведено исследование спектров пропускания в диапазоне частот 0.05-1.0 сыворотки крови крыс в динамике развития экспериментального рака печени. Были исследованы образцы сыворотки крови здоровых крыс и особей с привитыми опухолями через 14 и 28 дней после имплантации опухолевых клеток. Развитие данной патологии характеризуется статистически значимым снижением концентрации как общего белка, так и альбумина в крови. Показано, что THz спектры образцов от здоровых животных и после имплантации опухоли имеют подобную форму, но отличаются по амплитуде, что коррелирует с содержанием белка в образцах, определенного биохимически.

Анализ наблюдаемых отличий проводили путем сравнения полученных экспериментальных спектров с модифицированной трухкомпонентной релаксационной моделью для описания диэлектрической функции водного раствора. Оказалось возможным провести анализ характерных отличий для сыворотки крови в норме и при развитии патологии. Наблюдаемые изменения $\mathrm{THz}$ отклика объясняются образованием связанной воды вокруг молекул белка; в онкологических образцах количество белка и связанной воды меньше. Модельное описание диэлек- 
трической функции образцов от здоровых и экспериментальных животных (28 дней после имплантации опухоли) предсказывает максимальную чувствительность неинвазивной $\mathrm{THz}$ диагностики в районе $100-300 \mathrm{GHz}$. Данные по ТНz спектрам пропускания хорошо коррелируют с результатами рефрактометрических измерений в видимой и ИК областях, что может быть использовано как дополнительный метод построения дисперсионной зависимости в $\mathrm{THz}$ области. Потенциальное преимущество THz диагностики заключается в возможности неинвазивных и бесконтактных измерений, например при использовании спектроскопии отражения от кожи, слизистой оболочки полости рта или конъюнктивы глаза.

\section{Финансирование работы}

Работа выполнена при частичной финансовой поддержке гранта РФФИ 17-00-00275 (17-00-00270, 1700-00272) в медико-биологической части, гранта РФФИ 18-52-00040 в части обработки экспериментальных результатов, при поддержке Министерства науки и высшего образования в рамках выполнения работ по Государственному заданию ФНИЦ „Кристаллография и фотоника“ РАН в части развития $\mathrm{THz}$ техники.

\section{Соблюдение этических стандартов}

Все применимые международные, национальные и/или институциональные принципы ухода и использования животных были соблюдены.

\section{Конфликт интересов}

Авторы заявляют, что у них нет конфликта интересов.

\section{Список литературы}

[1] Smolyanskaya O.A., Chernomyrdin N.V., Konovko A.A., Zaytsev K.I., Ozheredov I.A., Cherkasova O.P., Nazarov M.M., Guillet J.P., Kozlov S.A., Kistenev Yu.V., Coutaz J.-L., Mounaix P., Vaks V.L., Son J.-H., Cheon H., Wallace V.P., Feldman Yu., Popov I., Yaroslavsky A.N., Shkurinov A.P., Tuchin V.V. // Progress in Quantum Electronics. 2018. V. 62. P. 1. doi 10.1016/j.pquantelec.2018.10.001

[2] Nazarov M., Shkurinov A., Tuchin V.V., Zhang X.-C. // Handbook of Photonics for Biomedical Science. Series in Medical Physics and Biomedical Engineering / Ed. by Tuchin V.V. CRC Press, Taylor and Francis Group, 2010. P. 519.

[3] Charkhesht A., Regmi C.K., Mitchell-Koch K.R., Cheng S., Vinh N.Q. // J. Phys. Chem. B. 2018. V. 122 (24). P. 6341. doi 10.1021/acs.jpcb.8b02872

[4] Reid C., Reese G., Gibson A.P., Wallace V.P. // IEEE J. Biomedical and Health Informatics. 2013. V. 17. N 4. P. 774.

[5] Fitzgerald A.J., Pickwell-MacPherson E., Wallace V.P. // PLOS ONE. 2014. V. 9. Issue 7. P. e99291.
[6] Ангелуи, А.А., Балакин А.В., Евдокимов М.Г., Есаулков М.Н., Назаров М.М., Ожсередов И.А., Сапожников Д.А., Солянкин П.М., Черкасова О.П., Шкуринов А.П. // Квант. электрон. 2014. Т. 44. № 7. С. 614.

[7] Yamaguchi S., Fukushi Y., Kubota O., Itsuji T., Ouchi T., Yamamoto S. // Scientific Reports. 2016. V. 6. P. 30124. doi 10.1038/srep30124

[8] Borovkova M., Khodzitsky M., Demchenko P., Cherkasova O., Popov A., Meglinski I. // Biomedical Optics Express. 2018. V. 9 (5). P. 2266. doi 10.1364/BOE.9.002266

[9] Chernomyrdin N., Gavdush A., Beshplav S.-I., Malakhov K., Kucheryavenko A., Katyba G., Dolganova I., Goryaynov S., Karasik V., Spektor I., Kurlov V., Yurchenko S., Komandin G., Potapov A., Tuchin V., Zaytsev K. // Proc. of SPIE. 2018. V. 10716. P. $107160 \mathrm{~S}$.

[10] Фундаментальная и клиническая физиология: Учебник для студ. высш. учеб. заведений / Под. ред. Камкина А.Г., Каменского А.А. М.: Издательский центр „Академия“, 2004. $1072 \mathrm{c}$.

[11] Tseng T.-F., You B., Gao H.-C., Wang T.-D., Sunet C.-K. // Opt. Express. 2015. V. 23. N 7. P. 9440.

[12] Gusev S.I., Balbekin N.S., Sedykh E.A., Grineva E.N., Khodzitsky M.K. // J. Phys.: Conference Series. 2016. V. 735 (1). P. 012088.

[13] Jeong K., Huh Y.-M., Kim S.-H., Park Y., Son J.-H., Oh S.J., Suh J.-S. // J. Biomed. Optics. 2013. V. 18 (10). P. 107008.

[14] Sun C.K., Chen H.Y., Tseng T.F., You B., Wei M.-L., Lu J.-Y., Chang Y.-L., Tseng W.-L., Wang T.-D. // Scientific Reports. 2018. V. 8. P. 3948. doi 10.1038/s41598-018-22060-y

[15] Gusev S.I., Demchenko P.S., Cherkasova O.P., Fedorov V.I., Khodzitsky M.K. // Chinese Optics. 2018. V. 11 (2). P. 182.

[16] Chen H., Chen X., Ma S., Wu X., Yang W., Zhang W., Liet X. // J. Infrared Millimeter and Terahertz Waves. 2018. V. 39. P. 399.

[17] Черкасова О.П., Назаров М.М., Ангелуи А.А., Шкуринов А.П. // Опт. и спектр. 2016. Т. 120. № 1. С. 59.

[18] Cherkasova O.P., Nazarov M.M., Smirnova I.N., Angeluts A.A., Shkurinov A.P. // Phys. Wave Phenomena. 2014. V. 22. N 3. P. 185.

[19] Назаров М.М., Черкасова О.П., Шкуринов А.П. // Квант. электрон. 2016. Т. 46. № 6. С. 488.

[20] Nazarov M.M., Cherkasova O.P., Shkurinov A.P. // J. Physics: Conference Series. 2017. V. 793. doi 10.1088/17426596/793/1/012005

[21] Nazarov M.M., Cherkasova O.P., Shkurinov A.P. // J. Infrared Millimeter and Terahertz Waves. 2018. V. 39 (9). P. 840.

[22] Shirshin E., Cherkasova O., Tikhonova T., Berlovskaya E., Priezzhev A., Fadeev V. // J. Biomed. Optics. 2015. V. 20 (5), P. 051033.

[23] Смолянская О.А., Кравценюк О.В., Панченко А.В., Одляницкий Е.Л., Гийе Ж.П., Черкасова О.П., Ходзиикий М.К. // Квант. электрон. 2017. Т. 47. № 11. С. 1031.

[24] Pan Q.-X., Su Z.-J., Zhang J.-H., Wang C.-R., Ke S.-Y. // Mol. Clin. Oncol. 2017. V. 6(4). P. 566.

[25] Назаров М.М., Шкуринов А.П., Ангелуи, А.А., Сапожников Д.А. // Известия вузов. Радиофизика. 2009. V. 52. Р. 595.

[26] Fukasawa T., Sato T., Watanabe J., Hama Y., Kunz W., Buchner R. // Phys. Rev. Lett. 2005. V. 95 (19). P. 197802.

[27] Назаров М.М., Шкуринов А.П., Кулешов Е.А., Тучин В.В. // Квант. электрон. 2008. Т. 38. № 7. С. 647. 
[28] Борн М., Вольф Э. Основы оптики. М.: Наука, 1973. 720 c.; Born M., Wolf E. Principles of Optics: Electromagnetic Theory of Propagation, Interference and Diffraction of Light. 7th Edition. Cambridge University Press, 1999. 987 p.

[29] Бучарская А.Б., Дихт Н.И., Абанасьева Г.А., Терентюк Г.С., Захарова Н.Б., Маслякова Г.Н., Хлебцов Б.Н., Хлебцов Н.Г. // Саратовский научно-медицинский журн. 2015. T. 11 (2). C. 107.

[30] International Guiding Principles for Biomedical Research Involving Animals (2012) CIOMS \& ICLAS.

http://www.cioms.ch/index.php/12-newsflash/227-ciomsand-iclas-release-the-newinternational-guiding-principles-forbiomedical-researchinvolving-animals.

[31] Cherkasova O.P., Nazarov M.M., Shkurinov A.P. // Optical and Quant. Electron. 2016. T. 48. N 3. C. 217.

[32] Bashkatov A.N., Berezin K.V., Dvoretskiy K.N., Chernavina M.L., Genina E.A., Genin V.D., Kochubey V.I., Lazareva E.N., Pravdin A.B., Shvachkina M.E., Timoshina P.A., Tuchina D.K., Yakovlev D.D., Yakovlev D.A., Yanina I.Yu., Zhernovaya O.S., Tuchin V.V. // J. Biomed. Optics. 2018. V. 23 (9). P. 091416.

[33] Sydoruk O., Zhernovaya O., Tuchin V., Douplik A. // J. Biomed. Optics. 2012. V. 17 (11). P. 115002. 